\title{
The application of wearable smart sensors for monitoring the vital signs of patients in epidemics: a systematic literature review
}

\author{
Niloofar Mohammadzadeh ${ }^{1} \cdot$ Marsa Gholamzadeh $^{2} \cdot$ Soheila Saeedi $^{2} \cdot$ Sorayya Rezayi $^{2}(0)$
}

Received: 15 July 2020 / Accepted: 27 October 2020

c) Springer-Verlag GmbH Germany, part of Springer Nature 2020

\begin{abstract}
Wearable smart sensors are emerging technology for daily monitoring of vital signs with the reducing discomfort and interference with normal human activities. The main objective of this study was to review the applied wearable smart sensors for disease control and vital signs monitoring in epidemics outbreaks. A comprehensive search was conducted in Web of Science, Scopus, IEEE Library, PubMed and Google Scholar databases to identify relevant studies published until June 2, 2020. Main extracted specifications for each paper are publication details, type of sensor, disease, type of monitored vital sign, function and usage. Of 277 articles, 11 studies were eligible for criteria. 36\% of papers were published in 2020. Articles were published in 10 different journals and only in the Journal of Medical Systems more than one article was published. Most sensors were used to monitor body temperature, heart rate and blood pressure. Wearable devices (like a helmet, watch, or cuff) and body area network sensors were popular types which can be used monitoring vital signs for epidemic trending. $65 \%$ of total papers $(n=6)$ were conducted by the USA, Malaysia and India. Applying appropriate technological solutions could improve control and management of epidemic disease as well as the application of sensors for continuous monitoring of vital signs. However, further studies are needed to investigate the real effects of these sensors and their effectiveness.
\end{abstract}

Keywords Wearable sensors $\cdot$ Vital signs monitoring $\cdot$ Epidemics outbreak $\cdot$ Body area network

\section{Introduction}

Health services due to information technology development have great changes, especially in remote monitoring (Li et al. 2019). Focusing on disease prevention and early detection of high-risk disease disabilities is one of the main goals of using physical sensor networks (Majumder et al. 2017). Todays, the smart systems and developed tools have significantly increased for the instant monitoring among the patients and controlling their conditions (Gries et al. 2018). The ability of such smart systems in storing and transferring data is of great importance in different branches of healthcare (e.g. Telemedicine) (Ha et al. 2018). Wearable systems

Sorayya Rezayi

s_rezayi@razi.tums.ac.ir

1 Department of Health Information Management, School of Allied Medical Sciences, Tehran University of Medical Sciences, Tehran, Iran

2 Health Information Management Department, School of Allied Medical Sciences, Tehran University of Medical Sciences, Tehran, Iran are mainly used for monitoring the symptoms and status of patients, follow up, telemedicine, monitoring of nursing and medical team systems, surgery robots, and many other systems (Lin et al. 2018; Majumder and Deen 2019). However, wearable sensors have received tremendous attention over the past decade, mainly concentrated in the healthcare industry. Such products attempt to apply physical signals such as heart rate, blood pressure, skin temperature, respiratory rate and body motion to extract clinically relevant information (Nag et al. 2019). Wireless body sensor networks (WBAN) include a number of heterogeneous biological sensors (Richesson et al. 2019). The sensors of such networks are placed on different areas of the body and these sensors can be worn or implanted on the body of the person. Each of these sensors requires specific requirements to identify and record symptoms (Rajan et al. 2018; Rezayi et al. 2019).

Since many diseases and disabilities require continuous monitoring in the present era, the continuity of patient monitoring for timely intervention seems essential (Dahiya et al. 2019; Richesson et al. 2019). As such, one of the most important areas of application of wearable technologies in healthcare field is utilizing WBANs to monitor patients. 
In epidemic outbreaks or under EMS (emergency medical service) conditions, patient monitoring is so sensitive and important (Ha et al. 2018). In these situations, the momentary monitoring of patients helps the medical team to take the necessary measures without delay. Patient monitoring announces the threatening events to caretakers, and most of such systems use the physiological input data for the direct control of support tools (Baskar et al. 2020; Kristoffersson and Lindén 2020). Anyhow, considering the present global conditions, the smart wearable sensors for patients s monitoring have the capacity and potential to be a major breakthrough in efforts to control the epidemics. An epidemic is defined by the Centers for Disease Control and Prevention (CDC) as a sudden increase in the number of cases of an infectious disease within a community or geographic area during a specific time period (Edoh 2019; Rahman et al. 2020).

Over the years, many outbreaks of infectious diseases have occurred and spread across the world (Kristoffersson and Lindén 2020). According to the points which were mentioned above, the way of public health surveillance could enlighten by using wearable device data. Since time and speed of operation in disease outbreaks control are so critical, patient monitoring (respiratory rate, temperature rate, blood pressure, ECG, heart rate, SPo2) in the prevalence of epidemics can be considered as an important phase for control and monitor real-time and accurate data (Zhu et al. 2020).

A riches of novel innovative technologies in the form of smart wearable technologies is achieving to greaten global precision and becoming accessible for the main aims of like preventing, monitoring and controlling the infectious diseases. The aim of this systematic review is provide a comprehensive investigation about applying wearable smart sensors for disease control and vital signs monitoring in infection epidemic outbreaks.

\section{Methods}

This systematic review was performed based on the Preferred Reporting Items for Systematic Reviews and MetaAnalysis (PRISMA) method (Moher et al. 2009). This method was introduced by Moher et al. and it is one of the best methods that authors can done their systematic reviews.

\subsection{Literature search}

In this review, a search of Web of Science, Scopus, IEEE Library, PubMed and Google Scholar databases was performed to identify relevant studies published until June 2, 2020.
The search strategy included a compressive combined keywords and mesh terms related to wearable smart sensors, patient monitoring and epidemics. The detailed search strategy for each database was presented in Table 1.

\subsection{Inclusion and exclusion criteria}

The academic selected papers were screened based on inclusion and exclusion criteria that have been displayed in Fig. 1.

\subsection{Data extraction phase}

In scientific searching 277 papers were retrieved based on some inclusion and exclusion criteria which were performed for selecting eligible articles. In this phase, the main classification of included articles was independently determined by three authors. These three authors (MG, SS and SR) synthesis the main characteristics and specifications of selected papers. They extracted main specification of papers. The next author (NM) evaluated the extracted information; she validated the main elements.

Three reviewers screened the abstract and full titles of papers independently. Another reviewer screened the selected studies randomly. These critical items are imported into a spreadsheet in Excel. The procedure of screening and selecting papers is presented in Fig. 2 based on PRISMA method. However, we presented the main characteristics and elements of included papers in Fig. 3.

\section{Results}

In searching databases phase, 277 relevant papers were retrieved. We removed duplicate studies, 178 citations remained. In the last phase of screening, only 11 relevant papers met our inclusion criteria. Our summary of papers is presented in Table 2. The author's main keywords for selecting papers are displayed by word cloud figure. In the presented figure, we demonstrate the main words with their importance weight; Fig. 4 is our word cloud schema.

\subsection{Illustration of papers}

\subsubsection{The distribution of papers based on countries and year of publication}

The included papers based on their country and year of publication are presented in Fig. 5. As we can see, from 11 included papers, four papers were published in 2020 by different countries and 3 papers were published in 2018. The remained papers were published from 2012 up to 2017. 
Table 1 Search strategy for each databases

\begin{tabular}{|c|c|}
\hline Database & Search strategy \\
\hline PubMed & $\begin{array}{l}\text { ("Wearable Electronic Devices"[MeSH Terms] OR "Wearable sensor" OR "Wearable device" OR "biosensor" OR "BAN" } \\
\text { OR "body sensor network" OR "BSN" OR "biomedical sensor" OR "IoT") AND ("disease Outbreaks"[MeSH Terms] OR } \\
\text { "Epidemics"[MeSH Terms] OR "Outbreak" OR "epidemic") AND ("monitoring, physiologic"[MeSH Terms] OR "Monitor- } \\
\text { ing, Physiologic" OR "Patient Monitoring" OR "Physiological Monitoring" OR "vital sign monitoring" OR "monitor" OR } \\
\text { "monitoring") } \\
\text { Results = 54 }\end{array}$ \\
\hline IEEE Library & 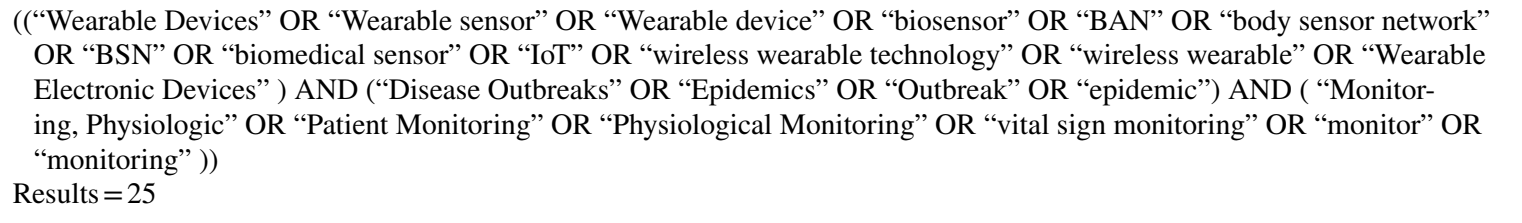 \\
\hline Web of Science & 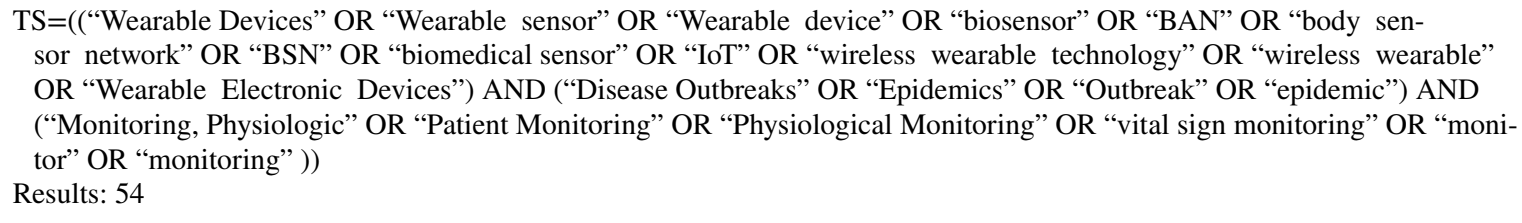 \\
\hline Scopus & 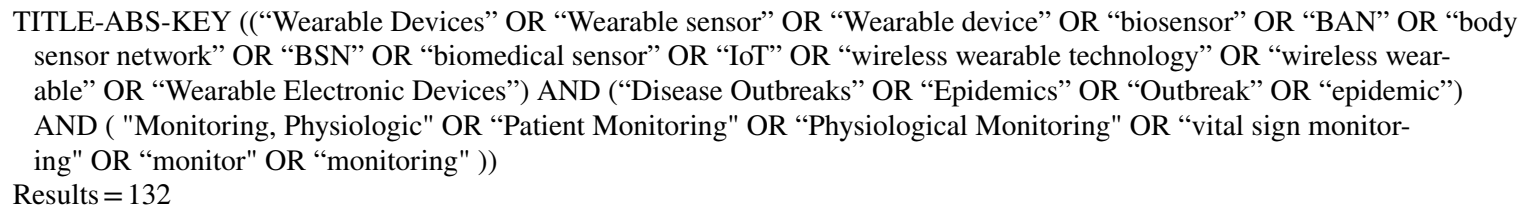 \\
\hline Google Scholar & $\begin{array}{l}\text { All in title: ("wearable sensor" OR "sensor" OR "BAN" OR “wireless sensor") AND (“epidemics" OR “outbreak disease” OR } \\
\text { "epidemic") } \\
\text { Results }=12\end{array}$ \\
\hline
\end{tabular}

Fig. 1 Exclusion and inclusion criteria to select eligible papers
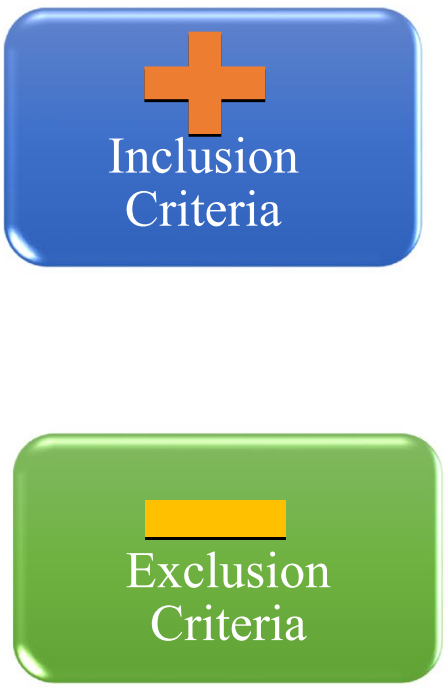

- Journal articles and proceedings

-Applying the wearable sensors in epidemics

- Mentioned the sensors type and related vital signs

-Sensors were used to monitor the patients

-Language: English
- Type of publication other than journal articles and proceedings (books, review papers, letters, etc.)

-The articles whose full text was not available in the English language

- Articles which was contained sensors not applied in epidemics outbreaks

\subsubsection{The distribution of included articles based on publisher names}

The selected papers $(n=11)$ were retrieved from 10 different journals. The total number of papers for each journal is displayed in Table 3. Two of 11 papers were published by Journal of medical systems. From these 11 included papers, none of them were published by conferences. 


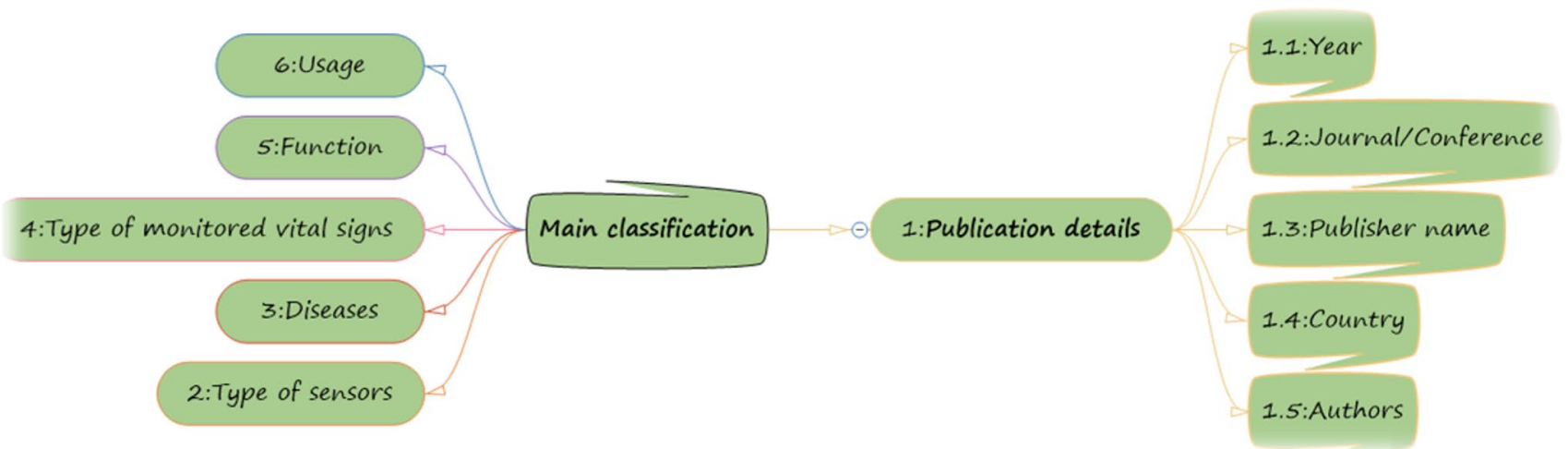

Fig. 2 Flow diagram of the literature search and study selection

Fig. 3 The main specifications of selected papers

\subsubsection{The distribution of monitored vital signs based on the wearable sensors}

The types of detected physiological vital signs based on the different wearable sensors is displayed in Table 4. As it is clear, wearable devices like a cuff or watch and wearable body area network sensors are popular for patient monitoring
Records identified through database

$$
\begin{aligned}
& \text { searching } \\
& (\mathrm{n}=277)
\end{aligned}
$$

PubMed $=54$, IEEE library $=25$, Web of

Science $=54$, Scopus $=132$ and Google Scholar $=12$

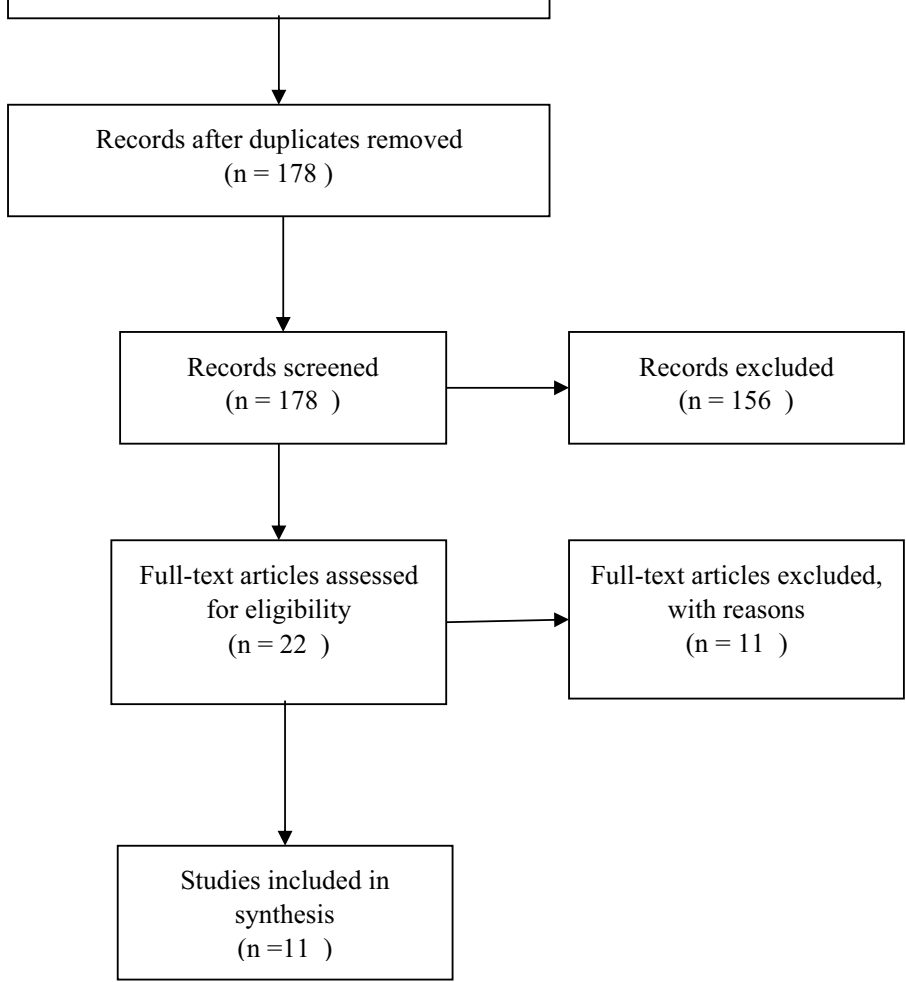

in epidemics controlling. However, the critical vital signs for patient monitoring in the mentioned condition is body temperature, heart rate and blood pressure. Table 3 is presented the details of this analysis. 


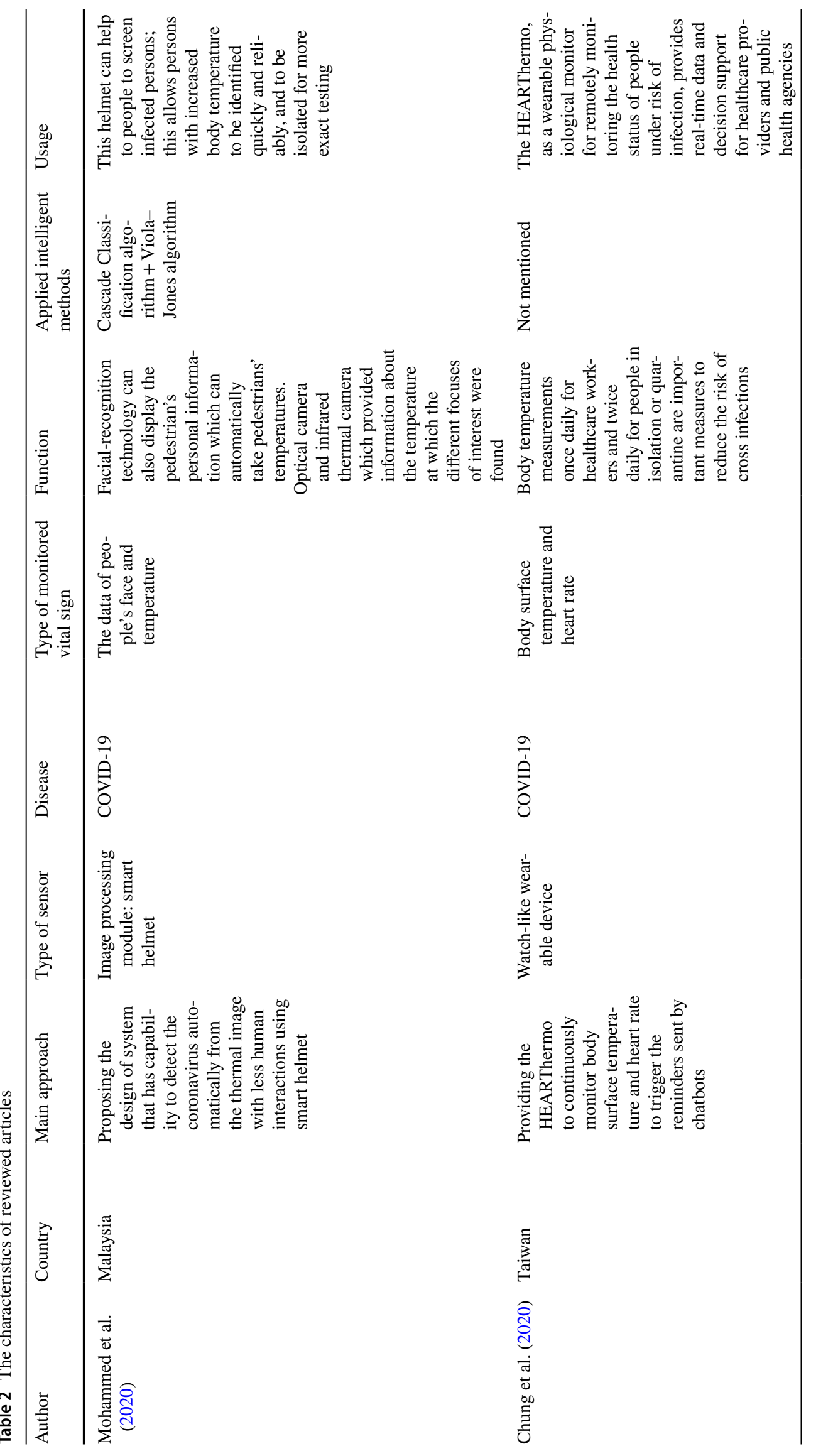




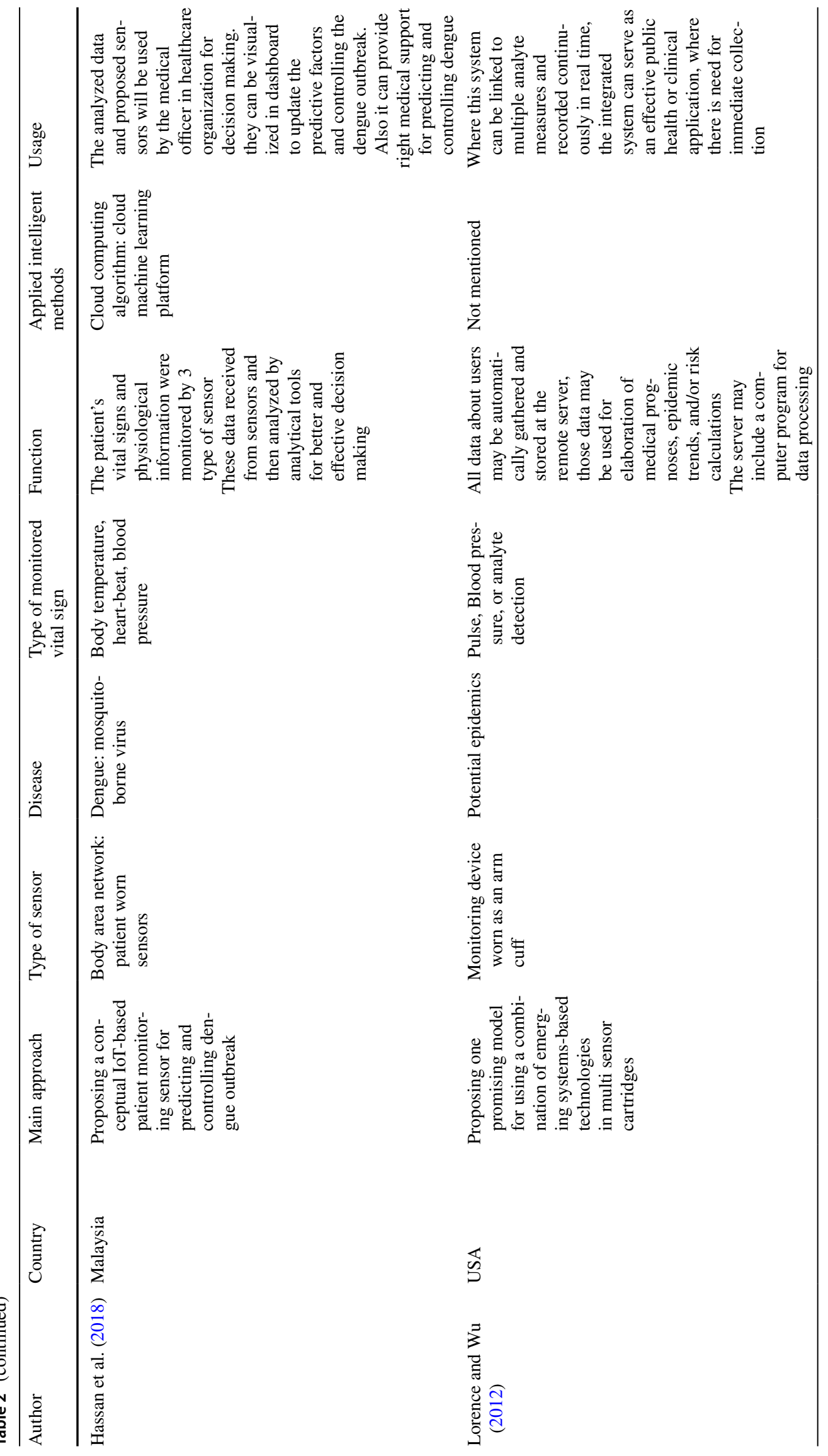




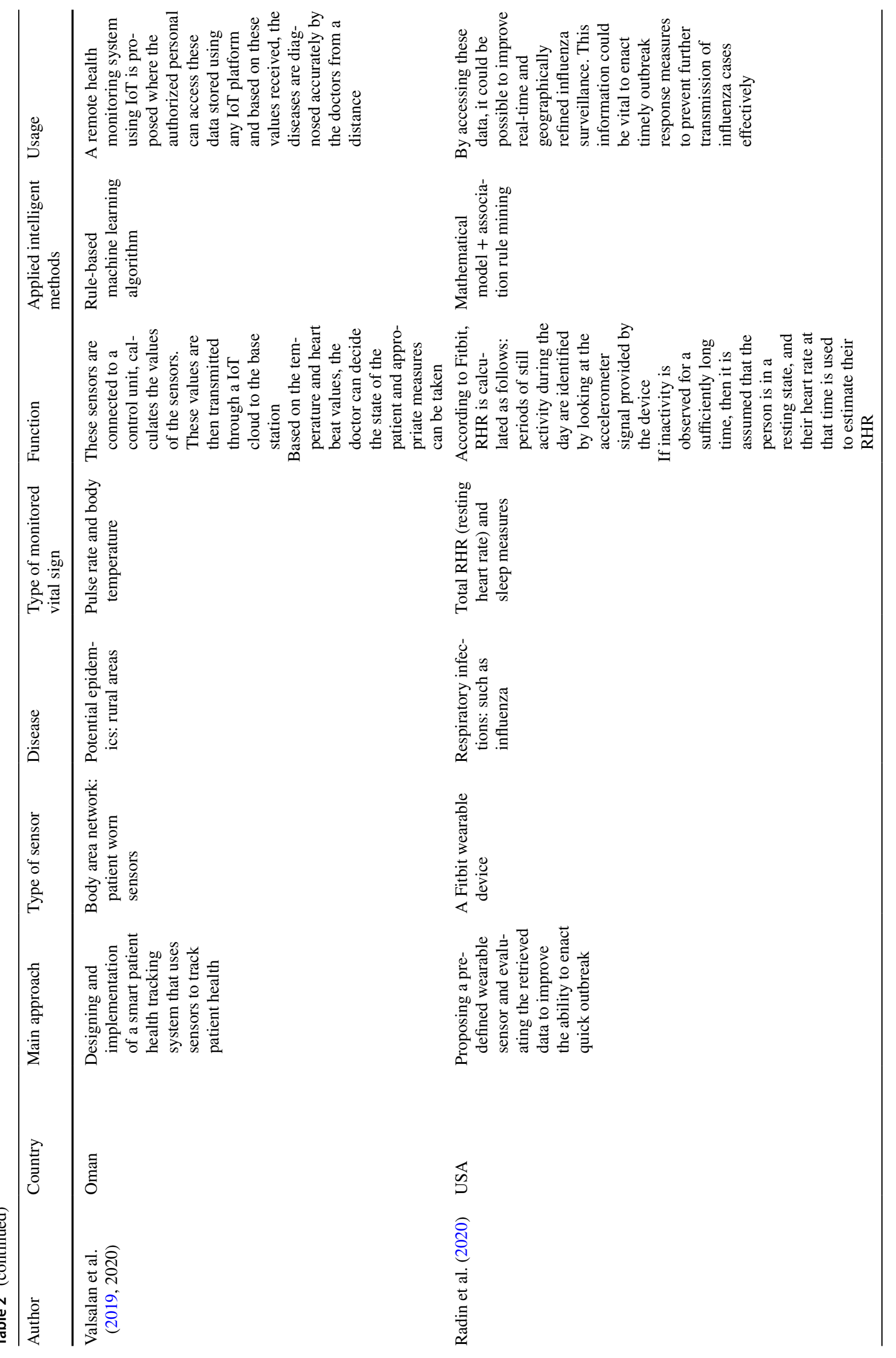




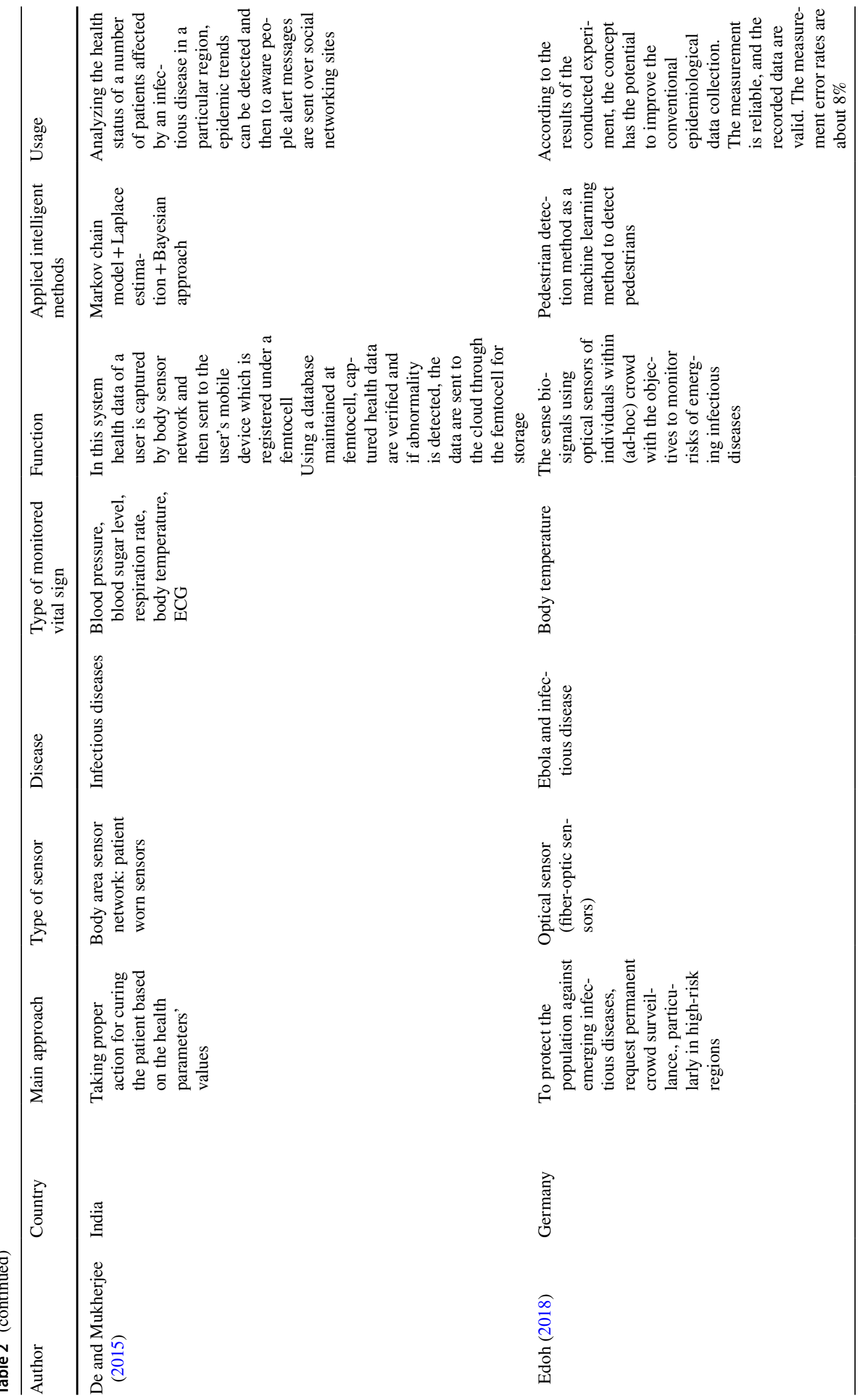




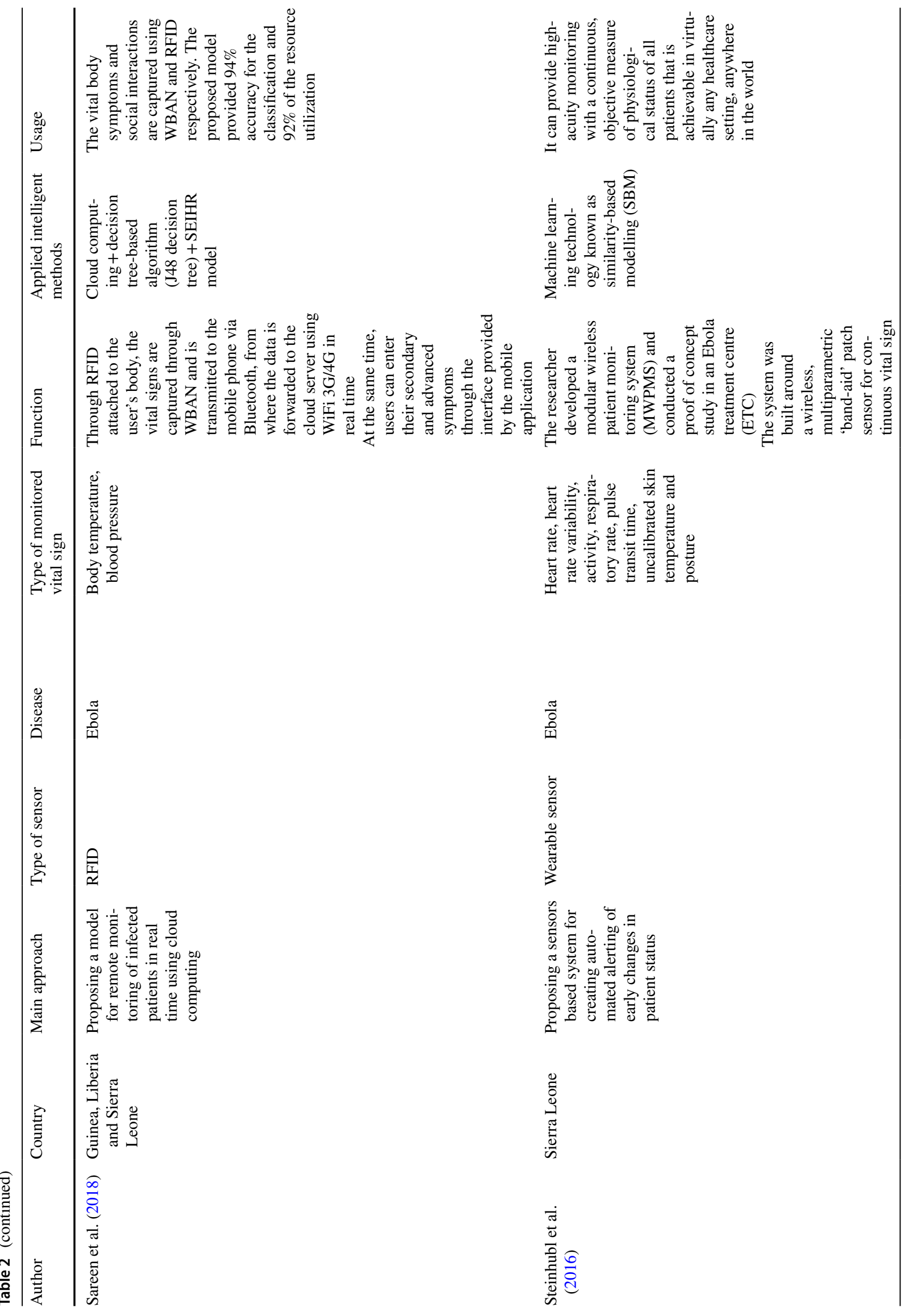




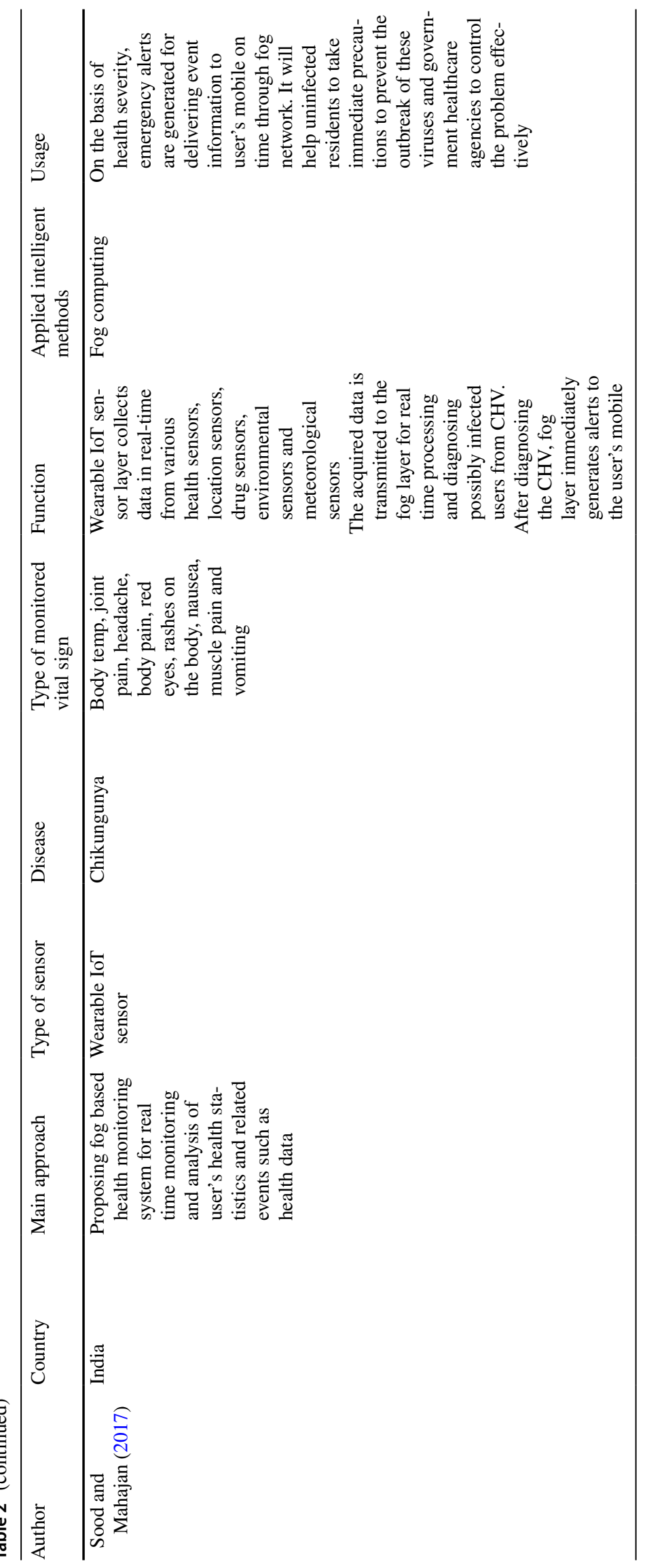

Springer 


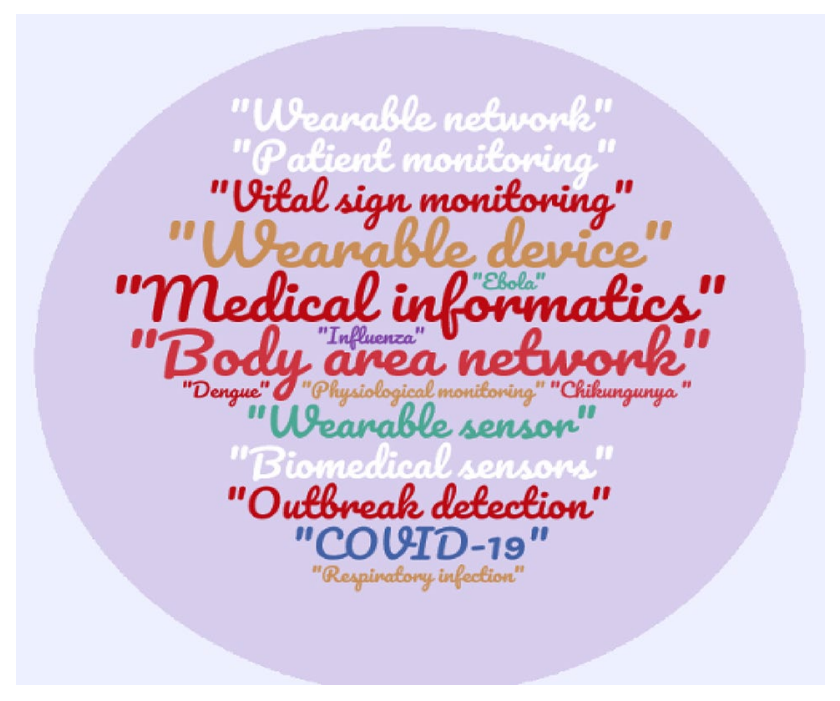

\subsubsection{The distribution of papers based on their main application and type of sensors}

In Fig. 6, as we can see wearable devices (like a helment, watch or cuff) are used for continuous monitoring and early detection. In addition, body area network sensors are a popular type which can used monitoring vital signs for epidemic trending. The details of each category are presented in mentioned figure.

\subsubsection{The distribution of papers based on countries}

The reviewed papers based on their counties is presented in Fig. 7. As it is clear, approximately $65 \%$ total papers $(n=6)$ were conducted by the USA, Malaysia and India. The other countries have relatively equal number of published articles.

Fig. 4 Word cloud of core keywords in included papers

Fig. 5 The distribution of articles based on countries and year

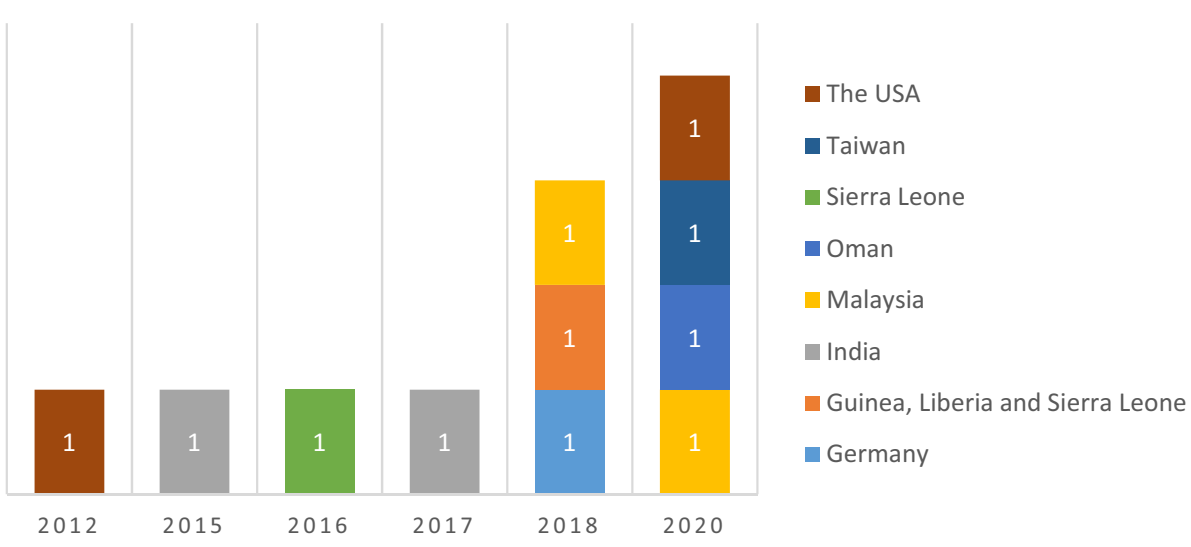

\subsubsection{The distribution of papers based on their disease and types of sensors}

\begin{tabular}{lc}
\hline Journal name & $\begin{array}{l}\text { Count of } \\
\text { papers }\end{array}$ \\
\hline Journal of Medical System & 2 \\
BMJ Global Health & 1 \\
Computers in Industry & 1 \\
International Journal of Grid and Distributed Computing & 1 \\
International Journal of Psychosocial Rehabilitation & 1 \\
Journal of Ambient Intelligent Humanized Computing & 1 \\
Journal of Critical Reviews & 1 \\
Journal of Medical Imaging and Health Informatics & 1 \\
Journal of Microbiology, Immunology, and Infection & 1 \\
The Lancet Digital Health & 1 \\
Grand total & 11 \\
\hline
\end{tabular}

Table 3 Distribution of studies based on their publishers
As we can see in drawn figure, wearable devices are appropriate technologies for patients with COVID-19. For Ebola epidemic RFID, wearable devices and optical sensors were used too. However, in Fig. 8 for each disease we can see applied different types of wearable sensors in detail respectively.

\subsubsection{The distribution of papers based on applied intelligent algorithms or Al oriented approaches}

In this section, we described the intelligent techniques and approaches used in the reviewed papers. Out of 11 citations, in 9 papers the applied AI oriented approaches were reported; most sensors designed to monitor patients in epidemic conditions (in reviewed papers) use intelligent methods to generate knowledge and information. But in 2 
Table 4 The distribution of monitored vital signs based on the wearable sensors

\begin{tabular}{|c|c|c|c|c|c|}
\hline Vital signs & BAN & RFID & Optical sensor & $\begin{array}{l}\text { Wearable } \\
\text { device }\end{array}$ & $\begin{array}{l}\text { Wearable } \\
\text { IoT sensor }\end{array}$ \\
\hline Body temperature & $* * *$ & $*$ & $*$ & $* * *$ & $*$ \\
\hline Hear rate & $* * *$ & & & $* * *$ & \\
\hline Blood pressure & $* *$ & $*$ & & & \\
\hline Facial-recognition & & & & $*$ & \\
\hline resting heart rate & & & & $*$ & \\
\hline Blood sugar level & $*$ & & & & \\
\hline Respiration rate & $*$ & & & $*$ & \\
\hline Myalgia & & & & & $*$ \\
\hline Red eyes & & & & & $*$ \\
\hline Rash & & & & & $*$ \\
\hline Neusea & & & & & $*$ \\
\hline
\end{tabular}

Fig. 6 The distribution of papers based on their main application and type of sensors

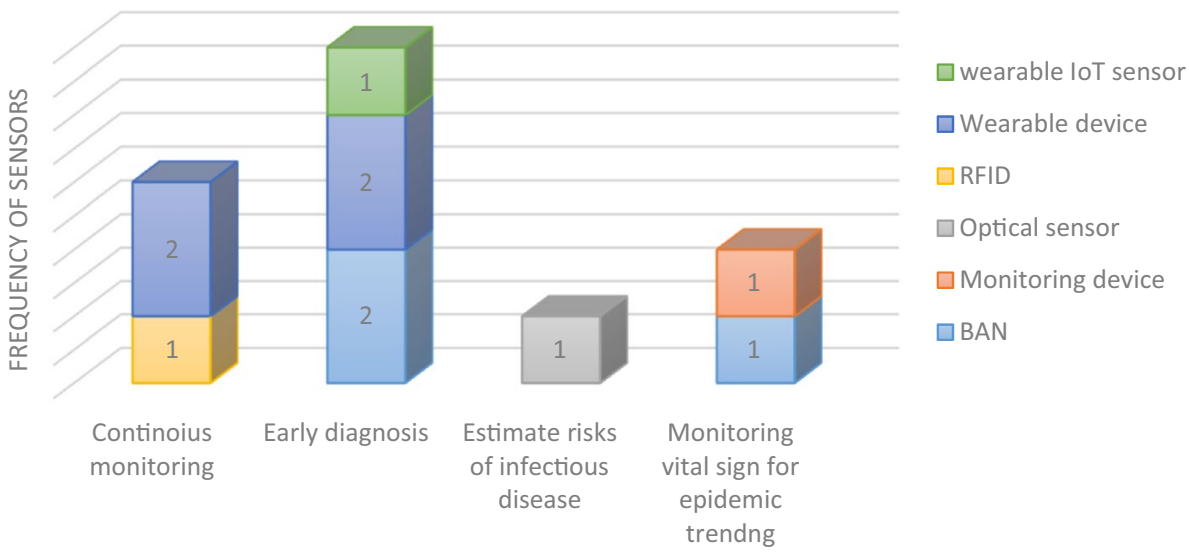

APPLICATION

artificial intelligence algorithms. Consequently, according to the reviewed articles, sensors will be able to raise the ability obtaining massive amounts of information or big data and enhance precision and accuracy. These sensors or mini devices also reflect the developmental tendency of modern sensors. Moreover, artificial intelligence is a novel stimulus for smart sensors, which gets sensors to think and learn and feed more efficient results back. Some of the applied algorithms by sensors or devices in papers are mathematical methods, rule-based algorithm, Bayesian approach, J48 decision tree and so on.

\section{Discussion}

According to our results, emerging wearable technologies have potential and capacity to control patients physiological vital signs in epidemic outbreaks. Wearable sensors are appropriate technologies that make easier continuous 
Fig. 8 The distribution of papers based on their disease and types of sensors

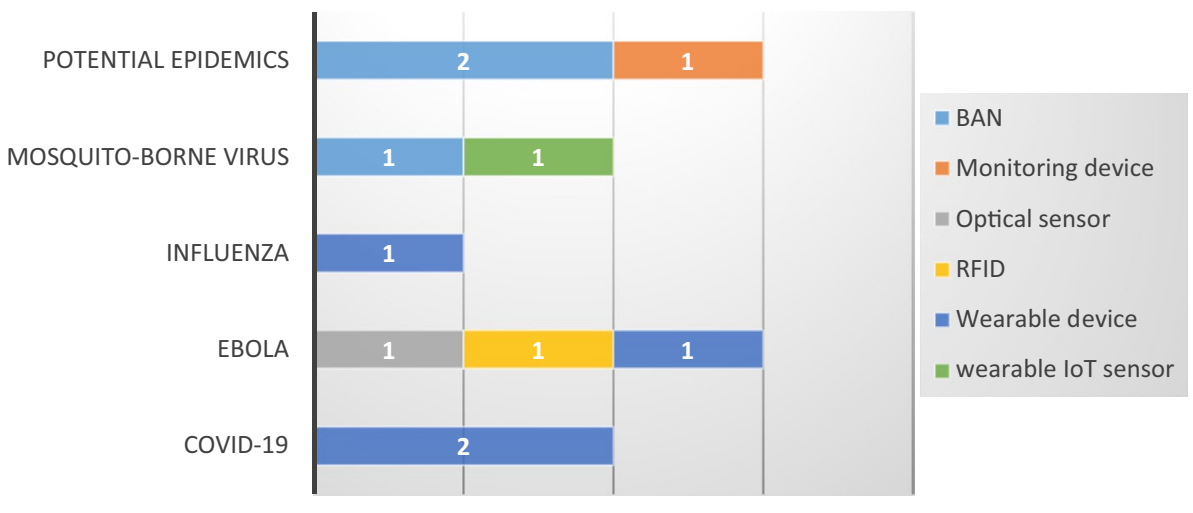

monitoring and control of the patients conditions for healthcare provider like physicians, nurses and specialists. The main objective of this review was identifying and analyzing the studies which conducted on the applying wearable sensors in epidemics outbreaks for patient monitoring.

According to studies, smart wearable sensors are designed to monitor patients in a variety of epidemics, such as Ebola, COVID-19 and Influenza. Contrary to researchers 'expectations, a small number of studies looked at the designing of wearable sensors to monitor vital signs such as body temperature, heart rate, blood pressure and a number of other studies to monitor patients' condition in the epidemics (Breteler et al. 2020). Anyway, Given that these tools can reduce the presence of patients outside the home due to the possibility of patient care at home and reduce patient traffic to the hospital and thus reduce interactions between people and also have great potential to identify, these tools are expected to be widely used in this area (Mohammed et al. 2020). Given the high cost of manufacturing and networking sensors, it can be argued that one of the main reasons for the low number of studies in this field is to provide funding for the construction of sensors and body networks (Dias and Paulo Silva Cunha 2018; Steinhubl et al. 2016).

Studies included in this review indicated that among communicable diseases, sensors were most applicable in infectious diseases. Such sensors could be applied for early diagnosis and continuous monitoring (Dias and Paulo Silva Cunha 2018). It is due to sensors have the potential to monitor and record vital signs of patients continuously and analyzed them. The most commonly used sensors in our study are wearable devices and BAN sensors. However, the evidence concerning the effectiveness of different types of smart sensors to control epidemic disease was almost inadequate.

As we expected, the majority of these devices recorded and monitored body temperature and heart rate as the most frequent vital signs for early detection and early response to stop the spread of communicable diseases (Chung et al. 2020; Edoh 2018; Mohammed et al. 2020; Radin et al. 2020; Sood and Mahajan 2017; Valsalan et al. 2019). In these type of smart devices, real-time data sent to healthcare providers simultaneously to track patients (Al-Janabi et al. 2017). Besides, with the new pandemic of COVID19 , researchers used wearable sensors for early detection of infected patients. In reviewed articles, two kinds of sensors were introduced (Chung et al. 2020; Mohammed et al. 2020). Researches applied wearable sensors to detect vital signs of patients, especially body temperature and respiratory rate. Consistent and accurate recording of vital signs in patients who suffered from COVID-19 is essential due to drastic changes in patients' signs and symptoms (Wang et al. 2020). The application of such sensors empowered clinicians for early diagnosis of new coronavirus to decrease its mortality. According to the positive outcomes of using sensors in similar pandemic diseases such as Ebola, it seems that using wearable sensors could be beneficial in patients who are hospitalized at home for remote vital sign monitoring to pass undesired changes to their physicians immediately.

The critical vital signs for patient monitoring in the epidemic disease in this review were body temperature, heart rate and blood pressure. Dias and Paulo Silva Cunha conducted a study of vital signs that are necessary for monitoring and concluded that heart rate, blood pressure, respiratory rate, blood oxygen saturation and body temperature are valuable vital signs (Dias and Paulo Silva Cunha 2018). These vital signs are essential for assessing a person's health status and can be used to identify clinical deterioration. In patients with COVID-19, fever is one of the most common symptoms in these patients (Tabata et al. 2020). Fever is one of the common clinical symptoms that appear when infectious diseases occur. It can be very helpful in diagnosing infectious diseases (Plaza et al. 2016). Heart rate is a standard vital sign that its monitoring can provide information about the body's physiological state (Plaza et al. 2016). Blood pressure monitoring is one of the most important indicators in determining a person's cardiovascular status (Dalvi et al. 2020). Wearable sensors can be used to monitor these vital signs and help determine the health status of patients and thus can be effective in diagnosing diseases, especially in measuring 
body temperature in infectious diseases and they can be very helpful to patients and health care providers.

The study had several strengths, including searches on various databases; Web of Science, Scopus, IEEE Library, PubMed, and Google Scholar. We also did not impose any time constraints on the article search process, and the articles presented at the conferences were also reviewed. The limitation of this study was to exclude non-English language studies.

\section{Conclusion}

This systematic review highlights the usage of different types of sensors to improve epidemic disease control. By applying a systematic approach, the authors provide a wide overview to use sensors that could monitor vital signs and disease progression through the epidemic. The survey showed that wearable sensors had the greatest potential in controlling and diagnosing the early signs of the disease in relation to epidemic diseases. Hence, applying appropriate technological solutions could improve control and management epidemic disease as well as the application of sensors for continuous monitoring vital signs. However, further studies are needed to investigate the real effects of these sensors and their effectiveness.

Funding In this paper, we didn't have any financial sponsor.

Availability of data and material All data generated or analyzed during this study are included in this published article.

\section{Compliance with ethical standards}

Conflict of interest The authors declare that there is no conflict of interest regarding the publication of this article.

Ethical approval This article does not contain any studies with human participants performed by any of the authors.

\section{References}

Al-Janabi S, Al-Shourbaji I, Shojafar M, Shamshirband S (2017) Survey of main challenges (security and privacy) in wireless body area networks for healthcare applications. Egypt Inform J $18: 113-122$

Baskar S, Shakeel PM, Kumar R, Burhanuddin MA, Sampath R (2020) A dynamic and interoperable communication framework for controlling the operations of wearable sensors in smart healthcare. Appl Comput Commun 149:17-26

Breteler MJM et al (2020) Vital signs monitoring with wearable sensors in high-risk surgical patients a clinical validation study anesthesiology. J Am Soc Anesthesiol 132:424-439
Chung Y-T et al (2020) Continuous temperature monitoring by a wearable device for early detection of febrile events in the SARS-CoV-2 outbreak in Taiwan, 2020. J Microbiol Immunol Infect 53:503

Dahiya AS et al (2019) Energy autonomous wearable sensors for smart healthcare: a review. J Electrochem Soc 167:037516

Dalvi C, Lamego MM, Kiani MJE, Poeze J, Vo H (2020) Blood pressure monitor with valve-chamber assembly. Google Patents

De D, Mukherjee A (2015) Femto-cloud based secure and economic distributed diagnosis and home health care system. J Med Imaging Health Inform 5:435-447

Dias D, Paulo Silva Cunha J (2018) Wearable health devices-vital sign monitoring, systems and technologies. Sensors 18:2414

Edoh T (2018) Risk prevention of spreading emerging infectious diseases using a hybrid crowd sensing paradigm, optical sensors, and smartphone. J Med Syst 42:91

Edoh TO (2019) Internet-of-things-enabled pre-screening for diseases: a novel approach for improving the conventional methodology and paradigm for screening for non-communicable diseases. In: Pre-screening systems for early disease prediction, detection, and prevention. IGI Global, pp 1-49

Gries A, Seekamp A, Wrede C, Dodt C (2018) Zusatz-Weiterbildung Klinische Akut-und. Notfallmedizin Deutschland Der Anaesth 67:895-900

Ha M, Lim S, Ko H (2018) Wearable and flexible sensors for user-interactive health-monitoring devices. J Mater Chem B 6:4043-4064

Hassan NH, Salwana E, Drus SM, Maarop N, Samy GN, Ahmad NA (2018) Proposed conceptual IoT-based patient monitoring sensor for predicting and controlling dengue. Int J Grid Distrib Comput $11: 127-134$

Kristoffersson A, Lindén M (2020) A systematic review on the use of wearable body sensors for health monitoring: a qualitative synthesis. Sensors 20:1502

Li J, Ma Q, Chan AHS, Man SS (2019) Health monitoring through wearable technologies for older adults: smart wearables acceptance model. Appl Ergon 75:162-169

Lin W-Y, Ke H-L, Chou W-C, Chang P-C, Tsai T-H, Lee M-Y (2018) Realization and technology acceptance test of a wearable cardiac health monitoring and early warning system with multi-channel MCGs and ECG. Sensors 18:3538

Lorence D, Wu J (2012) Computerized disease profiling using GPSlinked multi-function sensor cartridges. J Med Syst 36:2537-2545

Majumder S, Deen MJ (2019) Smartphone sensors for health monitoring diagnosis. Sensors 19:2164

Majumder S, Mondal T, Deen MJ (2017) Wearable sensors for remote health monitoring. Sensors 17:130

Mohammed MN, Syamsudin H, Al-Zubaidi S, Aks RR, Yusuf E (2020) Novel COVID-19 detection and diagnosis system using IOT based smart helmet. Int J Psychosoc Rehabil 24:7

Moher D, Liberati A, Tetzlaff J, Altman DG (2009) Preferred reporting items for systematic reviews and meta-analyses: the PRISMA statement. PLoS Med. https://doi.org/10.1371/journ al.pmed.1000097

Nag A, Mukhopadhyay SC, Kosel J (2019) Conclusion, challenges and future work printed flexible sensors. Springer, Cham, pp 193-198

Plaza JJG, Hulak N, Zhumadilov Z, Akilzhanova A (2016) Fever as an important resource for infectious diseases research. Intract Rare Dis Res 5:97-102

Radin JM, Wineinger NE, Topol EJ, Steinhubl SR (2020) Harnessing wearable device data to improve state-level real-time surveillance of influenza-like illness in the USA: a population-based study. Lancet Digit Health 2(2):e85

Rahman MS, Peeri NC, Shrestha N, Zaki R, Haque U, Ab Hamid SHA (2020) Defending against the novel Coronavirus (COVID-19) outbreak: how can the Internet of Things (IoT) help to save the world? Health Policy Technol 9:136-138. https://doi.org/10.1016/j. hlpt.2020.04.005 
Rajan K, Garofalo E, Chiolerio A (2018) Wearable intrinsically soft, stretchable, flexible devices for memories and computing. Sensors (Basel, Switzerland). https://doi.org/10.3390/s18020367

Rezayi S, Safaei AA, Mohammadzadeh N (2019) Requirement specification and modeling a wearable smart blanket system for monitoring patients in ambulance. J Med Signals Sens 9:234

Richesson RL, Andrews JE, Hollis KF (2019) Introduction to clinical research informatics. In Clinical research informatics. Springer, Cham, pp 3-15

Sareen S, Sood SK, Gupta SK (2018) IoT-based cloud framework to control Ebola virus outbreak. J Ambient Intell Humaniz Comput 9:459-476

Sood SK, Mahajan I (2017) Wearable IoT sensor based healthcare system for identifying and controlling chikungunya virus. Comput Ind 91:33-44

Steinhubl SR, Feye D, Levine AC, Conkright C, Wegerich SW, Conkright $\mathrm{G}$ (2016) Validation of a portable, deployable system for continuous vital sign monitoring using a multiparametric wearable sensor and personalised analytics in an Ebola treatment centre. BMJ Glob Health 1:e000070
Tabata S et al (2020) The clinical characteristics of COVID19: a retrospective analysis of 104 patients from the outbreak on board the Diamond Princess cruise ship in Japan. medRxiv:2020.2003.2018.20038125. https://doi. org/10.1101/2020.03.18.20038125

Valsalan P, Baomar TAB, Baabood AHO (2019) IOT based health monitoring system. J Crit Rev 7:2020

Wang S, Zhou X, Zhang T, Wang Z (2020) The need for urogenital tract monitoring in COVID-19. Nat Rev Urol 17:314-315. https://doi. org/10.1038/s41585-020-0319-7

Zhu G et al (2020) Learning from large-scale wearable device data for predicting epidemics trend of COVID-19. Discrete Dyn Nat Soc 2020:6152041. https://doi.org/10.1155/2020/6152041

Publisher's Note Springer Nature remains neutral with regard to jurisdictional claims in published maps and institutional affiliations. 